\title{
Extensão universitária: Impacto de um programa sob a perspectiva de promoção de saúde e formação profissional
}

\author{
University Extension: impact of a program from the perspective of health promotion and \\ vocational training
}

Extensión Universitaria: impacto de un programa bajo la perspectiva de promoción de salud y formación profesional

\author{
Júlia de Mattos ${ }^{\mathrm{I}}$, Daniel Pozzobon ${ }^{\mathrm{II}}$, Darcieli Lima Ramos ${ }^{\mathrm{III}}$, Luciane Sanchotene Etchepare \\ Daronco $^{\mathrm{IV}}$
}

\begin{abstract}
Resumo
O objetivo dessa pesquisa foi socializar os projetos e ações de um programa de extensão de uma Universidade Federal, bem como verificar qual público alvo impactado sob a perspectiva da promoção de saúde e formação profissional pelo programa de extensão NEMAEFS. Para tal, realizamos uma pesquisa do referido programa e cotejamos com o referencial bibliográfico que se debruça sobre a temática. Concluímos que os projetos e ações desenvolvidos pelo programa exercem impacto positivo à saúde da mulher e do trabalhador, aos processos de formação de estudantes de graduação e profissionais da área da saúde, bem como à comunidade.
\end{abstract}

Palavras-chave: Relações Comunidade-Instituição; Promoção da Saúde; Formação Profissional

\begin{abstract}
The aim of this research was to socialize the projects and actions of an extension program from a Federal University, as well as to verify which target audience was impacted from the perspective of health promotion and professional training through the NEMAEFS extension program. To this end, we conducted a survey of said program and compared it with the bibliographic reference that focuses on the theme. We conclude that the projects and actions developed by the program have a positive impact on the health of women and workers, on the training processes of undergraduate students and health professionals, as well as in the community.
\end{abstract}

Keywords: Community-Institutional Relations; Health Promotion; Professional Training

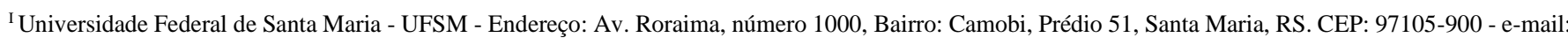
juliademattosufsm@gmail.com

II Universidade Federal de Santa Maria - UFSM - e-mail: danielpzb@gmail.com

III Universidade Federal de Santa Maria - UFSM - e-mail: darcielilimaramos@gmail.com

${ }^{\text {IV } U n i v e r s i d a d e ~ F e d e r a l ~ d e ~ S a n t a ~ M a r i a ~-~ U F S M ~-~ e-m a i l: ~ l u s a n c h o t e n e @ u f s m . b r ~}$
} 


\section{Resumen}

El objetivo de esta investigación fue socializar los proyectos y acciones de un programa de extensión en una Universidad Federal, así como verificar qué público objetivo fue impactado desde la perspectiva de promoción de la salud y formación profesional por parte del programa de extensión NEMAEFS. Para ello, realizamos un relevamiento de dicho programa y lo comparamos con la referencia bibliográfica que trata el tema. Concluimos que los proyectos y acciones desarrollados por el programa tienen un impacto positivo en la salud de las mujeres y los trabajadores, en los procesos de formación de estudiantes de grado y profesionales de la salud, así como en la comunidad.

Palabras clave: Relaciones Comunidad-Institución; Promoción de la Salud; Capacitación Profesional

\section{Introdução}

Nos últimos anos, a Extensão Universitária (EU) atingiu enorme avanço através do desenvolvimento de projetos em suas áreas temáticas específicas como saúde, cultura, meio ambiente, educação, direitos humanos, trabalho e comunicação (MOURA, 2015). De acordo com o Fórum de Pró-Reitores de Extensão Universitária das Instituições de Educação Superior Públicas Brasileiras (FORPROEX),

A extensão universitária, sob o princípio constitucional da indissociabilidade entre ensino, pesquisa e extensão universitária, é um processo interdisciplinar, educativo, cultural, científico e político que promove a interação transformadora entre Universidade e outros setores da sociedade. (FORPROEX, 2012, p.15).

Esse conceito contém em si as diretrizes básicas da EU e já vem sendo incorporado desde o estabelecimento da política de extensão da UFSM (UFSM; MINISTÉRIO DA EDUCAÇÃO, 2019). Nesse sentido, ainda há muitos desafios a serem superados, como a estimulação, por meio da EU, ao protagonismo estudantil no processo de mudança da educação superior, atualizar as áreas temáticas da EU, de forma a aumentar seu grau de consonância com os desafios contemporâneos e com as demandas inter e transdisciplinares, priorizar o desenvolvimento da EU enquanto produção de conhecimentos sistematizados, voltados para a emancipação dos atores nela envolvidos e da sociedade como um todo, contribuir para o desenvolvimento da ciência, da tecnologia e da inovação, com destaque para as tecnologias sociais produzidas na interação com a sociedade, visando à inclusão social e à melhoria das condições de vida (RENA, 2012). Desta forma, o fortalecimento da EU e de sua política carece de iniciativas e que estejam de acordo com o preceito constitucional de indissociabilidade entre Ensino, Pesquisa e Extensão. Para isso, é necessária uma articulação da EU com as políticas públicas, onde os efeitos positivos podem advir, primeiramente, de uma contribuição direta dos atores acadêmicos, por meio de suas ações extensionistas, na formulação, implementação e avaliação dessas políticas, especialmente as sociais, favorecendo, assim, o próprio fortalecimento das ações de extensão a elas vinculadas, em termos de financiamento, cobertura, eficiência e efetividade. Assim, a busca em promover a integração das ações 
extensionistas, assim como a das políticas públicas deve estar ancorada pelas diretrizes de interdisciplinaridade e interprofissionalidade (RENA, 2012).

Segundo Loch et al. (2020), em geral a formação em Educação Física (EF), e em outras áreas, ainda ocorre essencialmente na perspectiva uniprofissional em detrimento da integração de saberes e das práticas interprofissionais colaborativas. Nesse sentido, é entendido que o aprendizado e o trabalho interdisciplinar desde a formação inicial são fundamentais à transformação dos modos de organização do trabalho nos diferentes cenários de atuação profissional.

Nessa perspectiva, na tentativa de buscar uma formação que favoreça uma visão mais ampliada de saúde, integrar os saberes e práticas interprofissionais para seu fortalecimento, ampliar e aprofundar o conhecimento em medidas e avaliações, bem como fortalecer a prática extensionista na UFSM, surgiu, então, em 2001, o Núcleo de Estudos em Medidas e Avaliação dos Exercícios Físicos e Saúde (NEMAEFS), criado como um programa de extensão sob coordenação da Professora Doutora Luciane Sanchotene Etchepare Daronco e sendo certificado pelo Conselho Nacional de Desenvolvimento Cientifico e Tecnológico (CNPq) em 2007.

Inicialmente, o núcleo era composto apenas por acadêmicos do curso de EF, a partir de 2006, outros núcleos profissionais foram se inserindo. O NEMAEFS é focado em ações interdisciplinares, envolvendo além dos cursos de EF em licenciatura e bacharelado, outros cursos da área da saúde como Fisioterapia, Psicologia, Nutrição, Farmácia, Medicina, e demais interessados em temáticas relacionadas com a área de medidas e avaliação para EF e saúde, a fim de proporcionar um espaço de estudo e discussões aos diferentes profissionais e acadêmicos envolvidos.

O NEMAEFS tem por objetivos capacitar os profissionais e acadêmicos para realizar avaliações válidas, objetivas e fidedignas em seus alunos e pacientes nos diversos locais de atuação - academia, clube, escola, no trabalho personalizado ou no ambiente hospitalar - e proporcionar a prática de exercícios físicos para gestantes, pessoas com doenças crônicas degenerativas não transmissíveis (DCNTs), com distúrbios do desenvolvimento global, trabalhadores e acadêmicos.

A partir dos estudos, o Núcleo busca compreender conceitos da área - medidas, testes, objetividade e fidedignidade, conceitos básicos antropométricos, funcionais, metabólicos, neuromusculares, bem como métodos, técnicas e instrumentos para avaliação antropométrica em diferentes faixas etárias. Além disso, o NEMAEFS prepara os alunos para atuarem com pesquisa na área de medidas e avaliação da EF e saúde, na elaboração, condução e publicação dos projetos na forma de artigos científicos, além de proporcionar a acadêmicos e profissionais da área da saúde o conhecimento necessário para trabalhar exercícios físicos 
com pacientes portadores de doenças crônicas (diabetes, obesidades, asma e cardiopatias) e gestantes (UFSM, 2013a, 2019a).

O Núcleo incentiva os alunos à pesquisa, ensino e extensão, onde no decorrer desses anos conseguiu avaliar funcionários públicos, atletas de alto rendimento, estudantes, entre outros. Da mesma forma, disponibiliza cursos de capacitação para alunos, funcionários públicos e comunidade em geral sobre os conteúdos que tangenciam os saberes do núcleo. Nesse sentido, o reconhecimento das atividades de extensão como elos entre as atividades desenvolvidas nas universidades e a sociedade em geral é algo necessário e urgente.

O NEMAEFS é composto por acadêmicos de cursos da grande área da saúde, seja da UFSM ou outra instituição de ensino (IES), além de profissionais já graduados ou pós-graduados interessados em compreender e aplicar conhecimentos da área de Medidas de Avaliação. Em reuniões gerais são tratados assuntos comuns a todos os subgrupos de pesquisa e extensão. Cada subgrupo adota sua metodologia para se reunir e discutir as variáveis que serão estudadas a partir dos temas propostos, tanto na teoria como na prática, bem como a elaboração, desenvolvimento, coleta de dados, análise e posterior conclusão de pesquisas. Portanto, o objetivo deste trabalho é socializar os projetos e ações do programa de extensão NEMAEFS da UFSM, bem como verificar qual o público impactado sob a perspectiva da promoção de saúde e formação profissional pelo programa.

\section{A construção de projetos a partir do NEMAEFS}

O programa de extensão NEMAEFS direciona-se a profissionais, estudantes e comunidade em geral, desenvolvendo-se principalmente por meio de projetos de extensão, palestras e cursos. Dessa forma, o NEMAEFS realiza semestralmente o Workshop do Nemaefs, um evento gratuito, com diferentes temáticas em cada edição, sendo um evento importante na difusão e discussão de questões pertinentes à área da saúde, bem como na contribuição para o incremento desta linha de pesquisa da Ciência do Movimento humano e da saúde é formidável. O Workshop é considerado um incentivador e propagador de inovações e da promoção de atualização aos interessados nesta área de estudo, uma vez que os temas centrais escolhidos para os Workshops são oportunos e atuais, voltados ao crescimento técnico-científico possibilitando a atualização de profissionais e a construção de conhecimento de estudantes. Alguns temas abordados envolveram a atuação do personal trainer, saúde e inclusão, avaliação funcional, exercícios aquáticos e saúde, entre outros. O Workshop do Nemaefs atinge em torno de 100 participantes em cada 
edição, contemplando, desde sua primeira edição, em 2008, aproximadamente 1.800 ouvintes, envolvendo diversos profissionais, palestrantes, e possibilitando a troca de conhecimento.

Neste ano de 2020, o workshop do Nemaefs, em decorrência da pandemia do vírus Sars-CoV-2, causador da COVID-19, ocorreu pela primeira vez de forma totalmente online. Atingindo seus objetivos, esse evento proporciona em cada edição um espaço apropriado de estudo, debate e reflexão do conhecimento acerca de Exercícios Físicos e Saúde, possibilitando transposição deste conhecimento entre pesquisadores e ouvintes, expandindo o progresso cientifico desta temática (UFSM, 2020).

Outra construção do NEMAEFS foi Laboratório Interdisciplinar de Ergonomia e Cineantropometria (LABINTEC) criado em 2012 com o intuito de promover a prática das avaliações e medidas do ser humano por profissionais e acadêmicos dos cursos de Fisioterapia, EF, Nutrição, Psicologia e Medicina, preocupados com as temáticas referentes a saúde do trabalhador e saúde do escolar (UFSM, 2012). O LABINTEC serve de apoio as pesquisas do NEMAEFS e atualmente o laboratório envolve 65 estudantes de Graduação, 07 de Especialização e 03 de Mestrado acadêmico.

Em 2013, com o objetivo de ampliar os estudos acerca da educação física escolar, surgiu uma ramificação do NEMAEFS: O Núcleo de Estudos em Medidas e Avaliação da Educação Física Escolar. Esse grupo de estudos direciona-se à educação física escolar e sua avaliação, tanto qualitativa como quantitativamente. As linhas de estudos abordadas envolvem a avaliação da saúde e o aspecto pedagógico do ato de avaliar, considerando as temáticas: aptidão física e motora (qualidades físicas indicativas da saúde e atléticas), a interação social e afetiva, a proficiência motora, a inteligência corporal cinestésica, avaliação postural, exercícios físicos e doenças crônicas degenerativas, avaliação da dor, de lesões em estudantes e a ação pedagógica que envolve a "nota" nas aulas desta disciplina em diferentes níveis de ensino (UFSM, 2013b). Seguindo essa temática, o NEMAEFS ainda desenvolve o projeto de extensão Parceiros pela Educação Física Escolar Inclusiva (PEFEI), como meio de transformar as aulas de educação física escolar em uma porta para a inclusão escolar, através de oficinas nas escolas, cartilhas, palestras sobre esporte adaptado e inclusão, sobre evasão escolar na EF, doenças crônicas e exercícios físicos na escola, bem como aproximar os acadêmicos dos cursos de EF da UFSM das escolas públicas e privadas de Santa Maria - RS.

Ainda em 2013, criamos o "Grupo de Estudos da Dança: educação, arte e saúde”, o qual tem por finalidade promover encontros semanais práticos - workshops - para a preparação e execução de aulas de diferentes estilos de dança para todas as pessoas interessadas no ensino aprendizagem da dança e na dança como forma de educação para a saúde. As atividades são sempre ofertadas de forma gratuita, e ainda são realizados festivais e mostras de dança para a comunidade, envolvendo alunos de diferentes níveis de ensino e professores de dança e EF. Também conta com a participação de profissionais de outros cursos da área 
da saúde, como fisioterapeutas, psicólogos, médicos e nutricionistas para os encontros com fins de discussão científica. As linhas de pesquisa do grupo são: Saúde do escolar através da dança, Saúde do profissional da dança e Ensino aprendizagem da dança. Atualmente o grupo envolve 22 estudantes de Graduação, 06 de Especialização e 03 de Mestrado acadêmico.

Ao percebermos a necessidade de estudos acerca da avaliação e de orientações sobre exercícios físicos para gestantes e pessoas com DCNTs, bem como a carência de políticas públicas direcionadas a este público no município de Santa Maria, RS, e necessidade de formar profissionais da área da saúde capazes de trabalhar com esses grupos de forma multidisciplinar, criamos a partir do NEMAEFS o projeto de extensão: "Avaliações e orientações de exercícios físicos para pessoas com Doenças Crônicas Degenerativas Não Transmissíveis e gestantes da cidade de Santa Maria - RS.” É amplamente reconhecido que o exercício físico é benéfico para a diminuição do risco de desenvolvimento de inúmeras DCNTs, entre elas doenças cardiovasculares, diabetes mellitus tipo 2, e obesidade (COLBERG et al., 2010; DIAS; MONTENEGRO; MONTEIRO, 2014; NYSTORIAK; BHATNAGAR, 2018), bem como o exercício deve ser recomendado a mulheres grávidas por promover o bem-estar geral e ajudar a manter adequados tanto o ganho de peso gestacional quanto o ganho de peso fetal (GREGG; FERGUSON, 2017).

Nessa perspectiva, esse projeto de extensão tem por objetivo proporcionar através de avaliações periódicas e orientações sobre exercícios físicos, a promoção da saúde, prevenção de agravos, melhora da qualidade de vida e da funcionalidade de gestantes e pessoas com DCNTs desse município por profissionais e acadêmicos da área da saúde de forma multidisciplinar. A partir desse projeto de extensão é possível promover a melhora na qualidade de vida das pessoas com doenças crônicas e gestantes ao participarem dos exercícios físicos orientados pelo grupo de estudos. Em 2019, buscando ampliar a garantia de exercícios físicos a doentes crônicos e gestantes, o projeto "Pró Saúde" firmou parceria com a prefeitura municipal de Santa Maria, oferecendo sete modalidades - alongamento, caminhada, corrida, hiit, musculação, pilates e treinamento funcional - sendo ao todo 40 vagas por modalidade e todas ocorrendo no Centro Desportivo Municipal (CDM) da cidade. Além disso, o convênio entre a Prefeitura e a UFSM garante que os profissionais do Hospital Universitário de Santa Maria (HUSM) encaminhem os pacientes ao Pró Saúde.

Outro projeto que vai ao encontro desses objetivos é o NEMAEFS/HUSM, que visa qualificar profissionais da área da saúde a orientar exercícios físicos de forma multidisciplinar para públicos especiais em ambiente hospitalar, considerando a necessidade de maior aproximação entre a graduação e a saúde coletiva e a carência de políticas públicas direcionadas a esta ação no município de Santa Maria. Cabe 
destacar que esse projeto está sendo adaptado e em processo para ser trabalhado de forma virtual como “NEMAEFS/HUSM -Virtual” (UFSM, 2019c).

Por outro lado, preocupados com a saúde do trabalhador, criamos o projeto de extensão "Avaliação e orientação de exercícios físicos para policiais e guarda municipal da cidade de Santa Maria - RS”, dentro do Programa Saúde do Trabalhador e do Estudante, que realiza avaliações de saúde e integridade física e orientação à prática exercícios. O programa começou a ser elaborado em 2007, mas foi no ano de 2009 que passou a ser executado com a Polícia Civil, Militar e Federal, sendo a maior adesão às avaliações pelo do Batalhão de Operações Especiais (BOE). Até hoje, mais de três mil policiais do município já passaram por avaliações periódicas da postura, dor, lesões, aptidão motora e capacidade para o trabalho.

Todas as pessoas que realizam a avaliação preenchem uma ficha que fica no NEMAEFS e recebem uma apostila de avaliação com orientações de exercícios e esclarecimentos sobre doenças. A sala onde são realizadas as avaliações é privada, então o avaliado durante a avaliação é acompanhado apenas da presença do avaliador e pode escolher a ser avaliado por um homem ou uma mulher, sendo as avaliações são totalmente gratuitas.

Atuam no projeto acadêmicos e profissionais das áreas de EF, Fisioterapia, Medicina, Nutrição e Psicologia. O grupo lançou um livro intitulado "Medidas e Avaliações dos Exercícios Físicos e Saúde", com organização geral da professora coordenadora do projeto.

O NEMAEFS ainda conta com projetos institucionais. Assim, o projeto "De corpo e alma": exercícios físicos e qualidade de vida para servidores da UFSM tem como objetivo promover a melhoria na qualidade de vida e ampliar as relações sociais dos servidores ativos e aposentados da UFSM por meio da prática de exercícios físicos (UFSM, 2018b). Diversas modalidades têm sido ofertadas pelo projeto desde sua criação, em 2018, entre elas hidroginástica, natação, treinamento funcional e dança.

O projeto "De Corpo e Alma” oportuniza aos alunos da graduação dos cursos de EF e fisioterapia da UFSM a realização de estágios obrigatórios e profissionais dentro do campus da própria instituição de ensino, bem como gera pesquisas através da extensão, garantindo a indissociabilidade entre ensino, pesquisa e extensão, diretriz essa consolidada na Política de Extensão da UFSM (UFSM; MINISTÉRIO DA EDUCAÇÃO, 2019), contribuindo para o processo de formação dos estudantes. Os servidores periodicamente são avaliados de acordo com as seguintes variáveis: aptidão física, aptidão motora, funcionalidade, comportamento social e características antropométricas (UFSM, 2018a). A melhora do estresse, redução do sedentarismo, aumento da disposição para o trabalho, promoção de saúde e uma maior consciência corporal, bem como aumento da integração social são alguns dos diversos benefícios que o projeto é capaz de proporcionar aos seus participantes. 
Visando a saúde do trabalhador e reconhecendo que o ser humano, no desenvolvimento da sua função social, move e transforma a sociedade, bem como embasados em estudos que demonstram a existência de uma relação significativa entre saúde, aptidão física e desempenho no trabalho, atividades da vida diária e mobilidade (HANSEN et al., 2019; LEONG et al., 2015; SJØGAARD et al., 2016), criamos em 2017 o projeto institucional "CIA do Movimento - Exercícios Laborais na UFSM", como sucessor ao antes conhecido projeto de extensão Labor e Qualidade de Vida (LAQUAVI), de forma a oportunizar a prática de ginástica laboral pelos servidores da UFSM. A ginástica laboral é caracterizada por exercícios físicos coletivos, realizados por trabalhadores durante o expediente e dentro do ambiente de trabalho (SERRA; PIMENTA; QUEMELO, 2014), destinado a promover adaptações físiológicas, físicas e cognitivas contribuindo assim para a saúde e ajudando na diminuição do risco de desenvolvimento de doenças relacionadas ao trabalho.

O "CIA do Movimento" se propõe a promover melhorias nas condições fisiológicas, físicas e psíquicas por meio de exercícios físicos dirigidos, executados no ambiente de trabalho, aos servidores da UFSM. Através dele percebe-se a gama de benefícios proporcionados à saúde do trabalhador, entre eles o aumento da percepção corporal, disposição, autoestima e motivação. Percebemos uma grande melhora na coordenação, bem-estar, disposição, na percepção e consciência corporal dos servidores, bem como na motivação desses para a realização das tarefas cotidianas, contribuindo positivamente para uma melhor qualidade de vida no trabalho e no dia a dia dos adeptos ao projeto.

A Ginástica Laboral desenvolvida em grupos gera consequências positivas no desenvolvimento social como cooperação, socialização, solidariedade, liderança e laços de amizade, além de contribuir para a melhora na disposição de quem a pratica, tendo em vista que esses aspectos acabam impactando positivamente no cotidiano fora do ambiente de trabalho. Os dados coletados nas avaliações geram semestralmente trabalhos de conclusão de curso e monografias da pós-graduação, além de iniciação científica para acadêmicos.

Outro projeto de caráter institucional desenvolvido recentemente, em 2019, é o "ESPORTE UNIVERSITÁRIO: UFSM em Movimento" (UFSM, 2019b), oriundo da Direção do CEFD, o qual oferece inúmeras atividades esportivas como treinamento resistido e funcional, hidroginástica, natação, futebol, voleibol, lutas, para toda a comunidade acadêmica da UFSM. Em parceria com a Pró Reitoria de Extensão (PRAE) da UFSM, oferece semestralmente 30 bolsas para alunos monitores, atendendo mais de mil alunos da UFSM. Através do Esporte Universitário é possível promover práticas esportivas e de lazer aos estudantes, com o propósito de atender finalidades educativas, esportivas, de saúde e qualidade de vida, contribuindo para uma melhor saúde física e mental (PENEDO; DAHN, 2005), bem como oportunizando 
a integração social no âmbito da Universidade, interpessoal entre os cursos, (profissões acadêmicas), dos discentes e docentes dos diversos Centros da UFSM, por meio de variadas competições e outras formas de participação em eventos, envolvendo equipes e atletas, ou grupos e estudantes de Centros diversos. Na UFSM, propostas de EF aos universitários deixaram de existir absolutamente, uma vez que nenhuma iniciativa institucional com adesão voluntária foi proposta em substituição a prática obrigatória. Assim, o incentivo e desenvolvimento do hábito de um cuidado de si e, consequentemente, da saúde da comunidade acadêmica por meio das atividades que possibilitem uma maior integração intercursos e Inter centros é algo necessário e almejado pelo projeto (UFSM, 2017, 2019b). Casos de resoluções mais complexas e outras experiências inusitadas nos diferentes projetos, muitas vezes observadas durante os estágios, são levados para discussão em reuniões científicas realizadas mensalmente entre os alunos estagiários e a professora orientadora. Nessas reuniões são também apresentados e discutidos artigos científicos relativos à saúde, exercício físico, DCNTs, avaliação, além de se rediscutirem os objetivos do NEMAEFS, para que haja uma melhor atuação nos projetos, de forma sincronizada entre os professores e os estagiários. Ao todo, mais de duzentas pessoas entre profissionais e estudantes já fizeram parte em algum momento do núcleo desde sua criação.

O programa, que atende gratuitamente desde a sua implementação em 2001, já compartilhou experiências e contribuiu para a formação diferenciada de mais de 200 pessoas e possibilitou a pesquisa e conclusão de 141 trabalhos de conclusão de curso. Além disso, o programa possui 07 livros e 68 projetos de pesquisa publicados. Atualmente existem projetos que estão em processo de registro, como "Fatores antropométricos associados a obesidade", "Reabilitação Cardiovascular Domiciliar" e "Ativa Coração do RS”. Destacamos que todos os projetos de extensão e desenvolvimento institucional do NEMAEFS estão sendo adaptados e desenvolvidos de forma online, devido a pandemia da COVID-19.

\section{Considerações finais}

O programa de extensão universitária promovido pelo NEMAEFS se mostra uma excelente fonte de conteúdo para os envolvidos - estudantes, profissionais, comunidade. Os acadêmicos e profissionais que organizam e atuam nas ações adquirem conhecimentos através de estudos aprofundados e treinamentos específicos que, de outra maneira, não teriam acesso durante a graduação. O mesmo pode se dizer sobre as equipes de organização e avaliação das ações do programa, que aprendem a planejar e manter ativo esse tipo de programa ao nível institucional. E a comunidade se beneficia do programa através da participação 
gratuita em atividades planejadas, executadas e supervisionadas por pessoal qualificado cujo objetivo é melhorar a saúde e qualidade de vida dos participantes.

Portanto, é possível concluir que as ações e projetos desenvolvidos pelo programa estão alicerçados na tríade ensino, pesquisa e extensão, exercendo impacto positivo na saúde da mulher e do trabalhador, nos processos de formação de estudantes de graduação e aperfeiçoamento de estudantes de pós-graduação em EF, fisioterapia, psicologia, terapia ocupacional, medicina e farmácia, através da construção de conhecimentos, oportunizando o exercício da atuação em equipe multiprofissional, promovendo a interdisciplinaridade e proporcionando uma visão ampliada de saúde. Além disso, atinge ao público externo à UFSM, promovendo o entendimento da complexidade que representa o trabalho extensionista nos seus diferentes âmbitos e fortalecendo a relação entre universidade e comunidade.

\section{Referências}

COLBERG, Sheri R.; SIGAL, Ronald J.; FERNHALL, Bo; REGENSTEINER, Judith G.; BLISSMER, Bryan J.; RUBIN, Richard R.; CHASAN-TABER, Lisa; ALBRIGHT, Ann L.; BRAUN, Barry. Exercise and type 2 diabetes: The American College of Sports Medicine and the American Diabetes Association: Joint position statement. Diabetes Care, v. 33, n. 12, p. 2692-2696, 2010. DOI: 10.2337/dc10-9990.

DIAS, Ingrid; MONTENEGRO, Rafael; MONTEIRO, Walace. Exercícios físicos como estratégia de prevenção e tratamento da obesidade: aspectos fisiológicos e metodológicos. Revista Hospital Universitário Pedro Ernesto, Rio de Janeiro, v. 13, n. 1, p. 10, 2014. DOI: 10.12957/rhupe.2014.9808.

FORPROEX. Política Nacional de Extensão Universitária. Fórum de Pró-Reitores de Extensão das Universidades Públicas Brasileiras, Manaus, 2012.

GREGG, Vanessa H.; FERGUSON, James E. HANSEN, Gorm Mørk; MAROTT, Jacob Louis; HOLTERMANN, Andreas; GYNTELBERG, Finn; LANGE, Peter; JENSEN, Magnus T. Midlife cardiorespiratory fitness and the long-term risk of chronic obstructive pulmonary disease. Thorax, Copenhagen, v. 74, n. 9, p. 843-848, . Clinics in Sports Medicine, v. 36, n. 4, p. 741-752, 2017. DOI: 10.1016/j.csm.2017.05.005.

HANSEN, Gorm Mørk; MAROTT, Jacob Louis; HOLTERMANN, Andreas; GYNTELBERG, Finn; LANGE, Peter; JENSEN, Magnus T. Midlife cardiorespiratory fitness and the long-term risk of chronic obstructive pulmonary disease. Thorax, Copenhagen, v. 74, n. 9, p. 843-848, 2019. DOI: 10.1136/thoraxjnl-2018-212821.

LEONG, Darryl P. et al. Prognostic value of grip strength: Findings from the Prospective Urban Rural Epidemiology (PURE) study. The Lancet, London, v. 386, n. 9990, p. 266-273, 2015. DOI: 10.1016/S0140-6736(14)62000-6.

Loch, M.R., Rech, C.R., Costa. F.F. A urgência da Saúde Coletiva na formação em Educação Física: Lições com o COVID-19. Cien Saude Colet [periódico na internet] (2020/Jun). Disponível em: http://www.cienciaesaudecoletiva.com.br/artigos/a-urgencia-da-saude-coletiva-na-formacao-em- 
educacao-fisica-licoes-com-o-covid19/17618?id=1761.Acesso em: 08/09/2020.

MOURA, Denise Aparecida Soares De. A extensão universitária em história através da elaboração de instrumentos de pesquisa. Revista de Cultura e Extensão USP, São Paulo, v. 13, p. 97-107, 2015. DOI: 10.11606/issn.2316-9060.v13i0p97-107.

NYSTORIAK, Matthew A.; BHATNAGAR, Aruni. Cardiovascular Effects and Benefits of Exercise. Frontiers in Cardiovascular Medicine, v. 5, n. 135, 2018. DOI: 10.3389/fcvm.2018.00135.

PENEDO, Frank J.; DAHN, Jason R. Exercise and well-being: A review of mental and physical health benefits associated with physical activity. Current Opinion in Psychiatry, Miami, v. 18, n. 2, p. 189-193, 2005. DOI: 10.1097/00001504-200503000-00013.

RENA, Natacha. Urgente : é preciso fortalecer a extensão universitária. Revista de Extensão da UFMG, Belo Horizonte, n. 207, p. 8-17, 2012.

SERRA, Maysa Venturoso Gongora Buckeridge; PIMENTA, Lorrana Campos; QUEMELO, Paulo Roberto Veiga. Efeitos Da Ginástica Laboral Na Saúde Do Trabalhador. Revista Pesquisa em Fisioterapia, Franca, v. 4, n. 3, p. 197-205, 2014. DOI: 10.17267/2238-2704rpf.v4i3.436.

SJØGAARD, Gisela; CHRISTENSEN, Jeanette Reffstrup; JUSTESEN, Just Bendix; MURRAY, Mike; DALAGER, Tina; FREDSLUND, Gitte Hansen; SØGAARD, Karen. Exercise is more than medicine: The working age population's well-being and productivity. Journal of Sport and Health Science, Shangai, v. 5, n. 2, p. 159-165, 2016. DOI: 10.1016/j.jshs.2016.04.004.

UFSM; MINISTÉRIO DA EDUCAÇÃO. Política de Extensão da UFSM.Santa Maria, 2019.

UFSM. Portal de Projetos - Visualizar projeto. 2012. Disponível em: https://portal.ufsm.br/projetos/publico/projetos/view.html?idProjeto=39701. Acesso em: 10 jun. 2020.

UFSM. Portal de Projetos - Visualizar projeto. 2013a. Disponível em: https://portal.ufsm.br/projetos/publico/projetos/view.html?idProjeto=40616. Acesso em: 9 jun. 2020.

UFSM. Portal de Projetos - Visualizar projeto. 2013b. Disponível em: https://portal.ufsm.br/projetos/publico/projetos/view.html?idProjeto=41730. Acesso em: 10 jun. 2020.

UFSM. Portal de Projetos - Visualizar projeto. 2017. Disponível em: https://portal.ufsm.br/projetos/publico/projetos/view.html?idProjeto=58722. Acesso em: 9 jun. 2020.

UFSM. Portal de Projetos - Visualizar projeto. 2018a. Disponível em: https://portal.ufsm.br/projetos/publico/projetos/view.html?idProjeto=58998. Acesso em: 12 ago. 2020.

UFSM. Portal de Projetos - Visualizar projeto. 2018b. Disponível em: https://portal.ufsm.br/projetos/publico/projetos/view.html?idProjeto=60282. Acesso em: 10 jun. 2020.

UFSM. Portal de Projetos - Visualizar projeto. 2019a. Disponível em: https://portal.ufsm.br/projetos/publico/projetos/view.html?idProjeto=62936. Acesso em: 9 jun. 2020.

UFSM. Portal de Projetos - Visualizar projeto. 2019b. Disponível em: https://portal.ufsm.br/projetos/publico/projetos/view.html?idProjeto=63248. Acesso em: 10 ago. 2020. 
UFSM. Portal de Projetos - Visualizar projeto. 2019c. Disponível em: https://portal.ufsm.br/projetos/publico/projetos/view.html?idProjeto=64290. Acesso em: 10 jun. 2020.

UFSM. Portal de Projetos - Visualizar projeto. 2020. Disponível em: https://portal.ufsm.br/projetos/publico/projetos/view.html?idProjeto=66196. Acesso em: 10 jun. 2020.

\section{Como citar este artigo}

MATTOS, J; POZZOBON, D; RAMOS, D. L; DARONCO, L. S. E. Extensão universitária: impacto de um programa sob a perspectiva de promoção de saúde e formação profissional. Revista Kinesis, Santa Maria, Dossiê CEFD 50 anos, p.01-12, 2020.

*O presente trabalho não contou com apoio financeiro de nenhuma natureza para sua realização. 